Annls limnol. 9 (2) $1973: 177-182$.

\title{
UTILISATION D'UN VENTURI POUR UN ÉCHANTILLONNEUR DE DÉRIVE EN EAU COURANTE
}

\author{
par H. Décamps ${ }^{1}$, H. Laville ${ }^{1}$ et D. Trivellato ${ }^{2}$.
}

Un nouvel échantillonneur de dérive est décrit. il permet, au moyen d'un venturi et d'un manomètre différentiel, de connaître le débit d'eau filtré. Les conditions d'utilisation sont précisees.

\section{Use of a venturi meter for a drift sampler in running water}

A new drift sampler is described. It is possible, by means of a venturi tube and a differential manometer, to know the volume of water filtered. The conditions of utilization are specified.

Pour connaître la quantité d'eau ayant transité à travers un échantillonneur de dérive en eau courante, divers modèles comprennent un débitmètre (voir Flliott 1970).

Il s'agit le plus souvent d'un moulinet à hélice placé à l'avant ou à l'arrière d'un filet (Müller 1958, Elliott 1967). Un compteur indique le nombre de tours accomplis par l'hélice. Il est ainsi possible d'évaluer une vitesse moyenne du courant et, connaissant la section du filet, de calculer approximativement le débit filtré.

Cette solution n'est pas sans inconvénient pour de forts débits, notamment lors des mesures en période de crues. Placée à l'avant, l'hélice peut se détériorer ou abîmer les animaux en dérive; placée à l'arrière, elle ne donne des renseignements utiles que si le filet est enfermé dans un tube. De plus, la turbidité des eaux ne permet pas toujours de préciser à quel moment survient le colmatage du filet. Enfin, les compteurs enregistreurs de vitesse ne définissent pas toujours les variations dans le temps de la valeur mesurée.

1. Laboratoire d'Hydrobiologie, Université Paul-Sabatier, 118, route de Narbonne, 31077 Toulouse Cedex.

2. Institut de Mécanique des Fluides de l'ENSEEIHT, Laboratoire associé au C.N.R.S., 31 Toulouse. 
L'échantillonneur décrit dans cet article a été conçu plus précisément pour l'étude de la dérive des invertébrés lors du passage des crues.

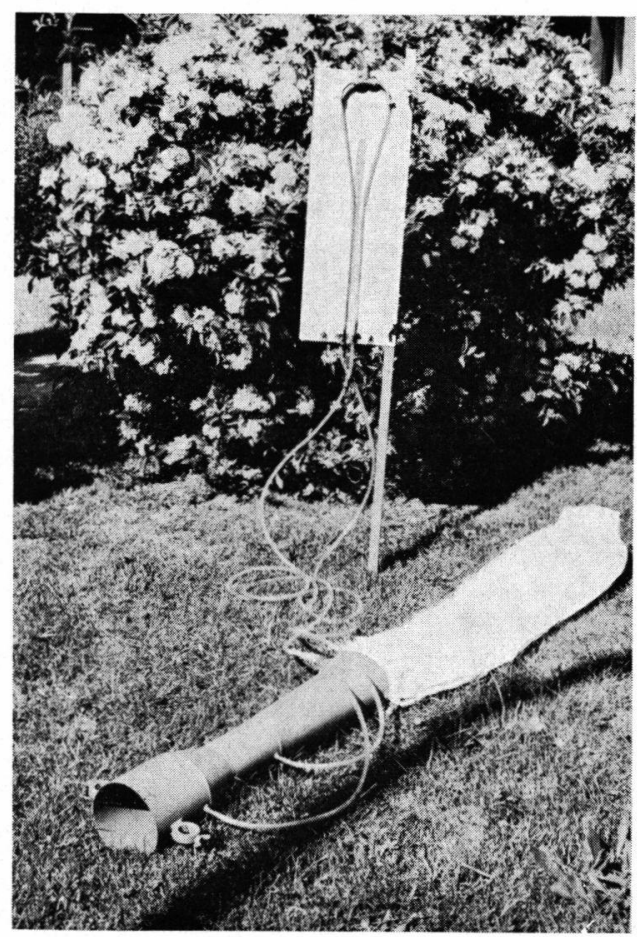

Fig. 1. - Vue générale de l'échantillonneur.

\section{L'échantillonneur.}

L'échantillonneur comprend essentiellement :

- le filet, disposé à l'aval, destiné au filtrage et à la récupération des invertébrés,

- l'appareil de mesure de débit, constitué d'un tube venturi métallique, muni de deux prises de pression A et B. Ces dernières sont reliées à un manomètre différentiel (fig. 2).

Le choix de cet appareil a obéi à plusieurs critères que nous pouvons résumer comme suit.

Dans une conduite en charge, un appareil déprimogène de ce type provoque une variation de pression mesurée supérieure à la perte de charge due à la mise en place de l'appareil. On peut donc penser, dans ce cas particulier, que la perte de charge à la traversée du tube sera réduite. D'autre part, l'effet bouchon produit 
par la présence d'un obstacle dans l'écoulement général de la rivière, et générateur de modifications notables de cet écoulement, ne sera sensiblement pas augmenté par rapport à celui produit par le filet de réception. Bien au contraire, la longueur de l'appareil permet à l'eau de recoller plus facilement à l'extérieur du tube et de réduire les perturbations aval.

L'application de la formule de Bernouilli dans un venturi permet d'obtenir l'équation suivante :

$$
\mathrm{Q}=\mathbf{K} \overline{\sqrt{\Delta h}}
$$

avec $\Delta \mathrm{h}=\mathrm{p}_{1}-\mathrm{p}_{2}$, différence de pression mesurée.

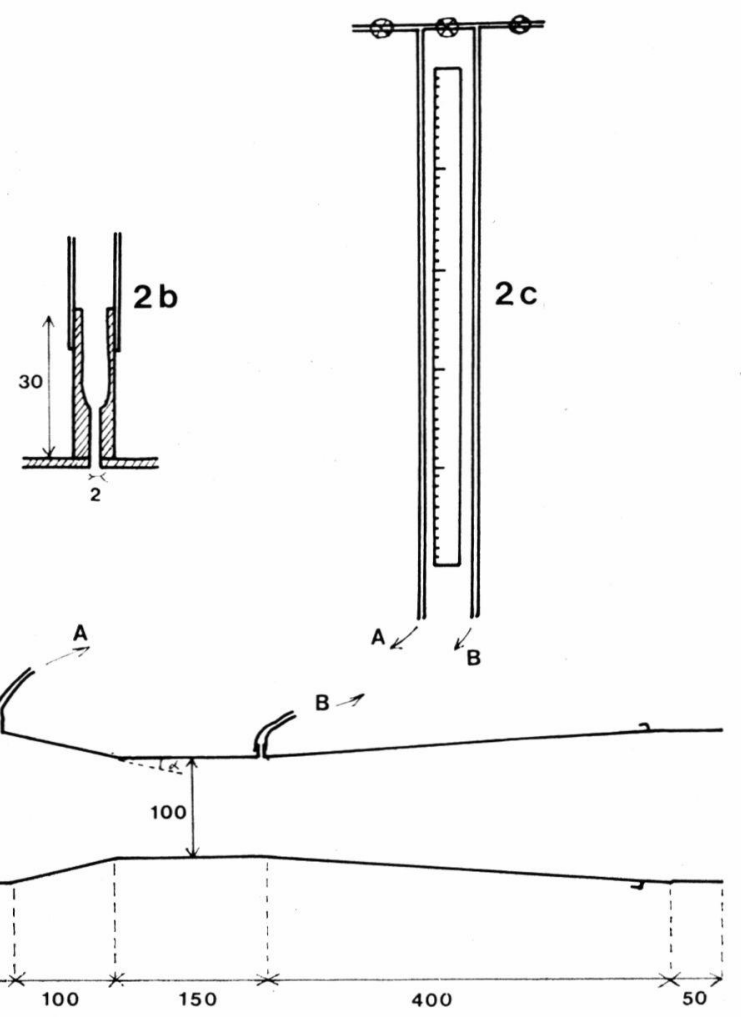

FIg. 2. - Schéma du venturi (2 a), d'une prise de pression (2 b) et du manomètre différentiel (2c).

Il est donc facile, après détermination du coefficient $\mathrm{K}$ de connaître le débit d'eau $Q$ traversant l'installation.

Des précautions indispensables sont à prendre lors de la réalisation ou de l'utilisation de l'appareil. A la construction, pour 
éviter ou réduire tout décollement à l'intérieur du tube, l'angle $\alpha$ doit être relativement réduit. A l'intérieur, la paroi ne doit pas présenter d'aspérités. Les arêtes ne doivent pas être agressives, mais adoucies.

Pour faciliter la mesure au manomètre différentiel en dehors du ruisseau, la partie supérieure des tubes piézométriques peut être fermée par un jeu de vannes ou de valves qui permettent la purge et le réglage du niveau d'eau dans le manomètre.

\section{Tarage.}

Pour déterminer la valeur du coefficient $\mathbf{K}$ dans I'équation $\mathbf{Q}=\mathbf{K} \sqrt{\Delta \bar{h}}$, nous avons procédé au tarage de l'appareil dans un laboratoire de l'Institut de Mécanique des Fluides de Toulouse. L'appareil était placé dans un canal dont le débit connu était réglable à volonté. Pour chaque valeur du débit, la différence de niveau $\Delta \mathrm{h}$ au manomètre était relevée. Sur la figure 3 , nous avons porté les points expérimentaux obtenus de $\Delta \mathrm{h}$ en fonction de $Q^{2}$ et tracé la droite moyenne. Il a donc été possible de déduire la constante $k$ des valeurs mesurées.

Sur 21 mesures, l'expérience a donné une valeur moyenne $k=3,85$. La formule de tarage du débitmètre est donc :

$$
\begin{array}{cc}
\mathrm{Q} & =3,85 \\
(\mathrm{l} / \mathrm{s}) & \sqrt{\Delta \overline{\mathrm{h}}} \\
(\mathrm{cm})
\end{array}
$$

\section{Conditions d'utilisation.}

Pour un fonctionnement correct, le tube venturi doit être entièrement immergé à l'amont et à l'aval. Cette condition est indispensable pour que la veine d'eau colle au divergent. De plus, il ne doit pas se former de vortex à l'amont.

L'appareil doit être placé dans la direction de l'écoulement. Nos essais ont montré qu'un angle de $7^{\circ}$ du tube avec cette direction ne provoquait pas de différence de mesure. Pour un angle de $10^{\circ}$, une erreur de $3 \%$ a été observée; elle peut être considéréc comme négligeable pour le sens à donner à nos expériences. La méthode du moulinet est beaucoup plus grossière et loin d'atteindre cette précision.

\section{Conclusion.}

A l'utilisation, cet appareil s'est révélé satisfaisant pour l'échantillonnage de la dérive du fond. Les variations du débit au cours de relevés horaires sont bien traduites par le manomètre 
différentiel enregistreur. Par ailleurs, lors de l'utilisation de mailles fines $(<110 \mu)$ le colmatage du filet est immédiatement décelé depuis la rive par simple lecture du manomètre.

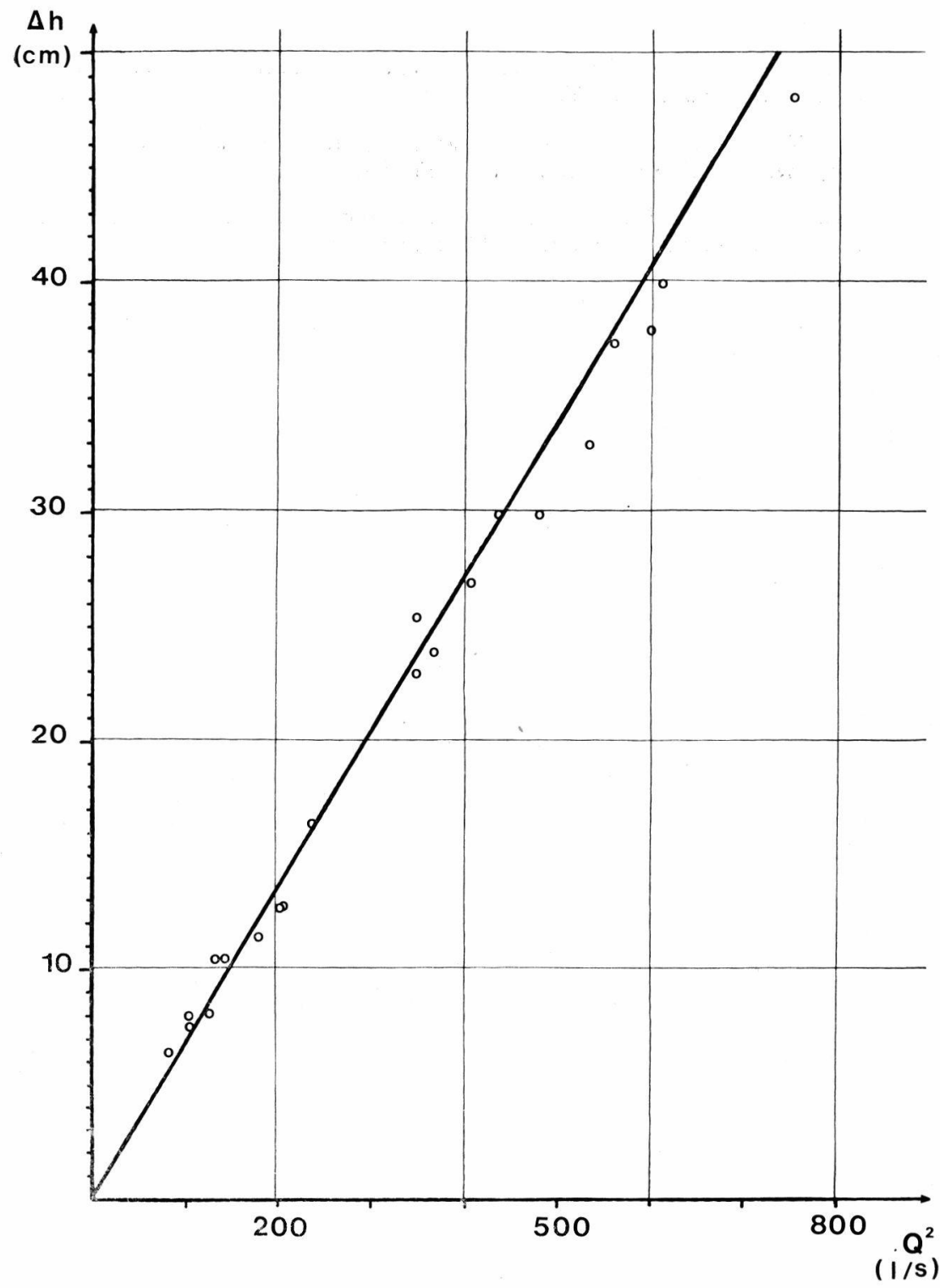

FIg. 3. - Points expérimentaux de $\Delta$ h en fonction de $Q^{2}$ et droite moyenne. 


\section{TRAVAUX GITÉS}

Elliott (J. M.). 1967. - Invertebrate drift in a Dartmoor stream. Arch. Hydrobiol., 63 : 202-237.

ElliotT (J. M.). 1970. - Methods of sampling invertebrate drift in running water. Annls Limnol., 6 : 133-159.

MüLLER (K.). 1958. - Beitrag zur Methodik der Untersuchung fliessender Gewässer. Arch. Hydrobiol., 54 : 567-570. 